\title{
Molecular investigation of the virucidal activity of proanthocyanidin from Alpinia zerumbet against the influenza A virus
}

Hayato Morimoto

RIBS, Okayama

Tadashi Hatanaka ( $\nabla$ hatanaka@bio-ribs.com )

RIBS, Okayama https://orcid.org/0000-0003-0325-3954

Mari Narusaka

RIBS, Okayama

Yoshihiro Narusaka

RIBS, Okayama

\section{Research}

Keywords: Alpinia zerumbet, influenza A virus, phytochemical, proanthocyanidin

Posted Date: October 18th, 2021

DOl: https://doi.org/10.21203/rs.3.rs-970727/v1

License: (c) (i) This work is licensed under a Creative Commons Attribution 4.0 International License. Read Full License 


\section{Abstract}

Proanthocyanidins (PACs) have various bioactivities, such as being anti-bacterial, anti-cancer, and antioxidant. Consequently, they have been vigorously studied for the development of new natural bioactive compounds. Recently, AzPAC was isolated from the medicinal plant Alpinia zerumbet, and it was found to inhibit the infection of animal viruses, influenza A viruses (IAVs), and porcine epidemic diarrhea virus. The virucidal activity of AzPAC means that it can interact directly with viral particles. However, few studies have investigated the preventive mechanism utilized by AzPAC on influenza virus replication. In this study, the composition of AzPAC and the affinity between AzPAC and IAVs was investigated in detail. We found that AzPAC was composed of an epicatechin monomer, which was linked by inter-flavan bonds between the $\mathrm{C} 4$ and $\mathrm{C} 8$ positions (B2-type) and the $\mathrm{C} 4$ and $\mathrm{C} 6$ positions (B5-type) in the terminal units of the PAC. A quenching assay indicated that AzPAC interacted with IAV membrane proteins, hemagglutinin and neuraminidase. Additionally, circular dichroism analysis indicated that AzPAC affected the change in the secondary structure rate of the viral membrane proteins. AzPAC was able to impair the infective process of IAVs via direct interaction with their viral membrane proteins. These results indicate that $A$. zerumbet is an invaluable bioresource for the development of preventive drugs against IAV infection.

\section{Introduction}

The three genera of influenza viruses A, B, C, and D in the Orthomyxoviridae family cause contagious respiratory problems that lead to annual epidemics and pandemics around the world (Asha and Kumar 2019), which in turn gives rise to significant increases in morbidity and mortality (Taubenberger and Morens 2008). Influenza A viruses (IAVs) in particular cause a virulent human infectious disease. IAVs have two viral membrane proteins, hemagglutinin (HA) and neuraminidase (NA), which are essential for host cell infection (Wagner et al. 2002; Edinger et al. 2014). HA recognizes the sialic acid receptors of the host cells at the early stages of the infection process, and subsequently, the viruses succeed in attaching to the cell surface and entering the host cells. In the late stages of the infection process, the sialic acids of the extracellular membranes are cleaved by NA, resulting in the release of progeny virus from the host cells and this facilitates the continuous infection of neighboring cells. Recently, the incorporation of HA and NA efficiently moved the IAVs on the surface of the host cells and enhanced the viral infection (Sakai et al. 2017). This showed that NA participates in the early stages of the infection process, in addition to the late stages of infection. Even though drugs targeting viral membrane proteins are being developed, the control of IAVs remains difficult because HA and NA cause frequent mutations and drug resistance (van de Wakker et al. 2017). Thus, the development of new alternative medicines is required.

Proanthocyanidins (PACs) belong to the family of polyphenols and have been attracting attention as phytochemicals because they possess various bioactivities (e.g., anti-lymphoma-tumor, anti-cancer, and anti-bacterial) (Zhang et al. 2005; Seiichi et al. 2019; Howell et al. 2005). Previous studies have reported that PAC oligomerized from flavan 3-ols, its monomeric components, and mixture extracts of the PACs and monomers have anti-animal-viral activity (Song et al. 2005; Derksen et al. 2014; Tsukuda et al. 2017; Luganini et al. 2018). Although natural products with virucidal compounds have been isolated from 
medical plants, the available species and tissue parts for use as bioresources for the extraction of bioactive compounds are limited.

Alpinia zerumbet, namely shell ginger, is a representative medical and herbal plant that grows in tropical and subtropical zones of Asia, including the Okinawa prefecture in Japan. It is used for traditional cuisine and in perfumes, whereas many shell gingers have little industrial use. Many medicinal components have been isolated from the plants in the Alpinia genus, including shell gingers, and they are recognized as traditional folk medicines (Ghosh and Ranga 2013). Narusaka et al. (2020) recently demonstrated that the extract from a mixture of leaves and pseudostems of $A$. zerumbet possessed antiviral activity against two plant-specific viruses, tomato mosaic virus (ToMV) and tobacco mosaic virus (TMV). Subsequently, polymetric procyanidin was isolated from an $A$. zerumbet extract and identified as an anti-plant viral molecule as well as an antiviral molecule against IAVs and porcine epidemic diarrhea virus (Hatanaka et al. 2021; Narusaka et al. 2021). However, detailed structures such as the end unit and terminal binding pattern of $A$. zerumbet-derived PAC (AzPAC), which are involved in the strong bioactivity of PAC, have not yet been determined. Additionally, the mechanism underlying the inhibitory process of IAV infection remains unknown.

The aim of this study was to clarify the compositional units of AzPAC and to investigate the binding affinity of PAC toward the IAV membrane proteins HA and NA. Moreover, the effect of AzPAC on the conformation of HA and NA was also assessed. These results have helped to elucidate the role of AzPAC in the prevention of IAV infection.

\section{Methods}

\section{Analysis of PAC composition by phloroglucinosis}

The leaves and pseudostems from A. zerumbet, polyphenol supplements originating from unripe fruit thinning of apple in the DHC Corporation (Tokyo, Japan), and the leaves of green tea (produced in Okayama, Japan) were used for PAC extraction. The extraction and confirmation of the weight-average molecular weight $(\mathrm{Mw})$ of the PAC were performed according to the procedure described by Hatanaka et al. (2021). The Mw of AzPAC was approximately 14,700, showing a larger Mw than that of the AzPAC previously extracted by Hatanaka et al. (2021). The PAC extract from apple (ApPAC) contained a polymeric $\mathrm{Mw}$. of approximately 8,400 as a major compound, in addition to two minor oligomeric compounds (Mw. approximately1700 and 400). Green tea-derived PAC (GtPAC) contains two major oligomers (Mw. approximately 1,900 and 1,200) (Narusaka et al. 2021). The production of 3-flavan-ols as a terminal unit was performed according to the procedures of Kennedy and Jones (2001). The lyophilized PAC (3 mg) was dissolved in $50 \mu \mathrm{L}$ of $\mathrm{dH}_{2} \mathrm{O}$, and then $500 \mu \mathrm{L}$ of phloroglucinol buffer $(0.1 \mathrm{~N} \mathrm{HCl}$ in 100 $\mathrm{mL}$ methanol containing $5 \mathrm{~g}$ phloroglucinol and $1 \mathrm{~g}$ ascorbic acid) was added. The dissolvent was divided into equal parts $(250 \mu \mathrm{L})$ into two microtubes. To confirm the presence of free 3-flavan-ol monomers, one of the two tubes was immediately neutralized with $250 \mu \mathrm{L}$ of $200 \mathrm{mM}$ sodium acetate. The other aliquot for the acid-catalyzed cleavage of PA was incubated at $50^{\circ} \mathrm{C}$ for $20 \mathrm{~min}$. The 
phloroglucinol reaction was stopped by adding $200 \mu \mathrm{L}$ of $200 \mathrm{mM}$ sodium acetate. The samples were centrifuged at $15,000 \mathrm{rpm}$ for $10 \mathrm{~min}$ at $25^{\circ} \mathrm{C}$. The supernatants were filtered using a $0.2 \mu \mathrm{m}$ membrane centrifugal filter (Merck Millipore, Darmstadt, Germany) for the HPLC system. The LC/MS analysis was performed using a Shimadzu HPLC system (Shimadzu, Tokyo, Japan) connected to the MS spectrum Amazon SL-OP (Bruker, Massachusetts, U.S.A.). The ionization was conducted using an electrospray ionization (ESI) source, and their mass spectra were detected in the negative ion mode. The injection sample $(1 \mu \mathrm{L})$ was subjected to HPLC using an Atlantis T3 column $(2.5 \times 100 \mathrm{~mm}, 3 \mu \mathrm{m}$; Waters, Manchester, UK) protected by a guard column containing the same material. The column was maintained at $40^{\circ} \mathrm{C}$. The separation was performed using a linear gradient of solvent $A\left(0.1 \%\right.$ formic acid in $\left.\mathrm{H}_{2} \mathrm{O}, \mathrm{v} / \mathrm{v}\right)$ and solvent $B$ (acetonitrile). At a flow rate of $0.2 \mathrm{~mL} \cdot \mathrm{min}-1$. The gradient parameter of the solvent $B$ concentration was as follows: 0-3 min, $1 \%$; 3-5 min, 6\%; 5-15 min, 18\%; 15-20 min, 55\%; 20-20.1 min, $1 \%$; 20.1-22 min, 1\%. Additionally, a washing step was performed to prevent carryover as much as possible. The flavan-3-ols and phloroglucinol adducts were detected based on the absorption at $280 \mathrm{~nm}$ and identified by co-chromatography using authentic chemical compounds and an MS spectrum. We identified the compounds by comparing their chemical properties with the following commercial standards using HPLC: (+)-catechin (Sigma-Aldrich, Tokyo, Japan), (-)-epicatechin (Nacalai Tesque, Inc., Kyoto, Japan), (-)-epicatechin-3-O-gallate, (-)-epigallocatechin (Tokyo Chemical Industry), (-)epigallocatechin (Tokyo Chemical Industry, Tokyo, Japan), procyanidin B1, procyanidin B2 (Extrasynthese, Genay, France), procyanidin B3 (ChemFaces, Wuhan, China), procyanidin B5 (Planta Analytica, New Milford, USA), chlorogenic acid (Sigma-Aldrich), neochlorogenic acid (Sigma-Aldrich), and 4-O-caffeoylquinic acid (Sigma-Aldrich). Phloroglucinol adducts for the monomeric epicatechin, monomeric catechin, and dimeric epicatechin were prepared from procyanidins B2, B3, and C1, respectively (Additional File 1: Figure S1).

\section{Fluorescence Quenching Assay}

The binding ability of PAC to the viral proteins was investigated using fluorescence spectroscopy based on the Stern-Volmer equation (Keizer 1983), as follows:

$$
\frac{F_{0}}{F}=1+K_{S V}[Q]
$$

$F_{0}$ and $F$ are the fluorescence intensities of the proteins in the absence or presence of the PAC quencher, respectively. $\mathrm{K}_{\mathrm{SV}}$ and [Q] are the Stern-Volmer quenching constant and a certain PAC concentration, respectively. NA influenza A H1N1 possessing the N295S mutation (Sino Biological Inc., A/California/04/2009) and the HA1 subunit (Sino Biological Inc., A/California/04/2009) were investigated using an interaction test with PAC. NA was dissolved in pure water according to the manufacturer's instructions and diluted 80 times with $1 \times$ PBS buffer $(\mathrm{pH} 7.4)$. The HA powder was dissolved in pure water, adjusted to a concentration of $0.25 \mathrm{mg} / \mathrm{mL}$, and subsequently diluted to a concentration of $0.085 \mathrm{mg} / \mathrm{mL}$ with $1 \times$ PBS buffer $(\mathrm{pH} 7.4)$. Fifty microliters of protein solution was 
added to the PAC solution ( $50 \mu \mathrm{L}$ ), and subsequently mixed gently in wells of black-colored multiple plates (Thermo Scientific, Nunc) and incubated at $25^{\circ} \mathrm{C}$ for $30 \mathrm{~min}$. The fluorescence intensity of NA was recorded between the emission spectrums of $275-380 \mathrm{~nm}$ with an excitation wavelength of $275 \mathrm{~nm}$; then, the intensity was subtracted from the fluorescence intensity of only the PAC solution. The excitation wavelength was $280 \mathrm{~nm}$ for the fluorescence quenching of $\mathrm{HA}$, and the emission spectrums were 280$410 \mathrm{~nm}$. Measurements were performed at $25^{\circ} \mathrm{C}$.

\section{Circular Dichroism Spectrum}

PAC lyophilizates were diluted with $0.1 \times$ PBS buffer $(\mathrm{pH} 7.4)$, and subsequently the concentration of the solutions was adjusted to $0.2 \mathrm{mg} / \mathrm{mL}$. The original NA solution was diluted with $0.1 \times$ PBS 50 times, and then used for $\mathrm{CD}$ analysis. HA lyophilizate $(100 \mu \mathrm{g})$ was diluted with $400 \mu \mathrm{L}$ of distilled water, and the concentration was adjusted to $0.83 \mathrm{mg} / \mathrm{mL}$ using $0.1 \times$ PBS. The viral protein solution $(200 \mu \mathrm{L})$ was added to microtubes containing $50 \mu \mathrm{L}$ of the PAC solution. The mixture was maintained at room temperature for $30 \mathrm{~min}$. After the reaction, the mixture was placed in a $1.00 \mathrm{~mm}$ cuvette. The CD spectrum was measured using a Chirascan CD Spectrophometer CW-3000 (Applied photophysics, Leatherhead, UK) at a wavelength range of $180-350 \mathrm{~nm}$ with $1.0 \mathrm{~nm}$ of step size and maintained at $25^{\circ} \mathrm{C}$ with a bandwidth $0.5 \mathrm{~nm}$, time-per-point $0.1 \mathrm{~s}$. The measurements were repeated five times. The obtained spectrum values were subtracted from the values of the background and averaged. The estimated secondary structure was calculated by combining the deep neural networks (CDNN) algorithm.

\section{Results And Discussion}

\section{Characterization of anti-viral PAC derived from A. zerumbet}

Previously, the structure of AzPAC (more than 40 DP degree of polymerization) from A. zerumbet was investigated using a DMAC assay and mechanical analyses with MALDI-TOF-MS and ${ }^{13} \mathrm{C}-\mathrm{NMR}$ (Hatanaka et al. 2021). The results indicated that AzPAC consisted of an epicatechin unit in a B-type carbon-carbon bond. However, the terminal binding pattern and end unit of AzPAC remain unknown. Here, phloroglucinosis clarified the composition of AzPAC and the binding pattern of flavan 3-ols. Epicatechinphloroglucinol was detected, and the extension unit of AzPAC was epicatechin (Fig. 1a). The procyanidin B2-phloroglucinol and two unknown procyanidin B-type-dimer-adducts were detected, and the C4-C8 bond occupied over half of the extension pattern. After phloroglucinosis, procyanidins B2 and B5 were detected in $73.1 \%$ and $26.5 \%$ of cases, respectively, and procyanidin B1 was detected in trace amounts. The results indicated that the C4-C8 inter-flavan bond almost occupied the terminal pattern, followed by the C4-C6 inter-flavan bond. These results showed that more than $99 \%$ of the end unit in AzPAC was epicatechin, and the terminal structure of AzPAC showed B2 and B5 linkage patterns (Fig. 1b). ApPAC was formed of only epicatechin in the extension unit and consisted of $75 \%$ epicatechin and $25 \%$ catechin in the terminal units (Fig. 1c). This result was similar to the ratio of catechin and epicatechin as terminal units in the PAC-rich fraction extracted from apple fruit skin previously (Mendoza-Wilson et al. 2016). Additionally, 
chlorogenic acid was detected in phloroglucinosis of AzPAC, but this compound originally contained in ApPAC extract (data not shown). The oligomeric PAC extracts isolated from green tea leaves (GtPAC) were predominantly comprised of 2,3-cis stereochemistry flavane-3-ol, which accounted for over $90 \%$ of the total terminal units, and epicatechin and epigallocatechin 3-gallate accounted for $20 \%$ and $41.8 \%$, respectively (Fig. 1d). The extension units of GtPAC consisted of $40.8 \%$ and $53.0 \%$ catechin and epicatechin, respectively. The (epi)gallocatechin-phloroglucinols were estimated to be extension units by MS analysis. Previously, Punyasiri et al. (2004) indicated that PAC isolated from tea leaves possessed gallocatechins as extension units. Thus, these results suggested that GtPAC had (epi)gallocatechin as a minor unit in the extension of PAC. In this study, the terminal patterns of ApPAC and GtPAC could not be determined because these PAC extracts contained substantial amounts of procyanidin dimers.

\section{Interaction of Azpac With Viral Membrane Proteins}

The affinity between PACs and IAV membrane proteins was analyzed using a quenching assay. Both AzPAC and ApPAC decreased fluorescence from HA and NA in a dose-dependent manner, whereas the addition of GtPAC to the viral proteins caused a gentle decrease in fluorescence (Fig. 2a and c). Additionally, the top peak of the fluorescence of HA shifted toward the red spectrum in the presence of a high concentration $0.16 \mathrm{mg} / \mathrm{mL}$ of AzPAC. The blue shifts were clearly confirmed in the NA in the presence of all types of PAC, suggesting a change in the NA conformation. This change in the NA conformation may be related to the anti-IAV activities of AzPAC, ApPAC, and GTPAC that were previously reported by Narusaka et al. (2021). The $\mathrm{K}_{\mathrm{sv}}$ values of AzPAC against both HA and NA were significantly higher than those of ApPAC and GtPAC (Fig. 2b and d). These results indicated that AzPAC has a higher affinity for the two viral membrane proteins when compared with ApPAC and GtPAC.

The $C D$ spectrum of the proteins in the presence of PAC was also evaluated to understand the change in the secondary structure of the viral membrane proteins, in addition to the binding affinity of PAC against viral proteins. When AzPAC and ApPAC interacted with HA, the rates of the secondary structures changed, but not GtPAC. In particular, the interaction of AzPAC and ApPAC with the viral proteins preferentially influenced the rate of a-helix and random coil formation. When AzPAC interacted with HA, the rate of ahelix a in the secondary structures of HA decreased by $2.0 \%$ and was accompanied by a $2.4 \%$ increase in the random coil rate (Table 1 and Fig. 3a). On the other hand, when ApPAC interacted with HA, the rates of a-helix and random coil increased or decreased by $1.8 \%$ and $1.1 \%$, respectively. Additionally, the rate of $\beta$ sheet antiparallel in HA decreased by $0.9 \%$ in the presence of ApPAC. The rate of a-helix and NA decreased or increased by $3.2 \%$, accompanied by a $2.4 \%$ increase in the rate random coil in the presence of AzPAC (Table 2 and Fig. 3b). The interaction of ApPAC with NA changed the rate of the a-helix in NA from $82.3-84.5 \%$. An increase of $1.1 \%$ in the rate of the a-helix of NA was observed after the addition of GtPAC. The change in the rate of a-helix in NA after the addition of the three PACs may be attributed to the blue shift in NA in the presence of the three PACs in the quenching assay. 
Table 1

Hemagglutinin secondary structures in the presence of AzPAC, ApPAC and GtPAC.

\begin{tabular}{llllll} 
Sample & \multicolumn{3}{l}{ Percentage of the hemagglutinin $(\mathrm{HA})$ secondary structures } \\
\cline { 2 - 6 } & a-helix & $\boldsymbol{\beta}$-sheet antiparallel & $\begin{array}{l}\boldsymbol{\beta} \text {-sheet } \\
\text { parallel }\end{array}$ & $\beta$-turn & Random coil \\
\hline HA free & 39.3 & 8.7 & 6.1 & 17.0 & 20.1 \\
\hline HA+AzPAC & 37.3 & 9.2 & 6.6 & 17.1 & 22.5 \\
\hline HA+ApPAC & 41.1 & 7.8 & 5.8 & 16.9 & 19.0 \\
\hline HA+GtPAC & 39.5 & 8.5 & 6.1 & 17.0 & 20.1 \\
\hline
\end{tabular}

AzPAC, Alpinia zerumbet-derived PAC; ApPAC, immature apple fruit-derived PAC; GtPAC, green teaderived PAC.

Table 2

Neuraminidase secondary structures in the presence of AzPAC, ApPAC and GtPAC.

\begin{tabular}{|llllll|}
\hline Sample & \multicolumn{5}{l}{ Percentage of the neuraminidase (NA) secondary structures } \\
\cline { 2 - 6 } & a-helix & $\boldsymbol{\beta}$-sheet antiparallel & $\boldsymbol{\beta}$-sheet parallel & $\boldsymbol{\beta}$-turn & Random coil \\
\hline NA free & 82.3 & 0.1 & 1.9 & 9.6 & 6.2 \\
\hline NA+AzPAC & 79.1 & 0.2 & 2.4 & 9.8 & 8.6 \\
\hline NA+ApPAC & 84.5 & 0.1 & 1.8 & 9.1 & 5.8 \\
\hline NA+GtPAC & 83.4 & 0.1 & 1.8 & 9.4 & 5.8 \\
\hline $\begin{array}{l}\text { AzPAC, Alpinia } \\
\text { derived PAC. }\end{array}$
\end{tabular}

Relationship between PAC structure and the inhibition of viral infection

The mean degree of polymerization of the PACs from the kiwifruit pericarps affected both the binding ability with tyrosinase and the efficiency of tyrosinase inhibition (Chai et al. 2014), suggesting that the degree of polymerization is important for bioactivity. This study also suggested that the high polymerization degree of PAC is related to the strength of the affinity against the IAV-derived proteins and the anti-IAV activity, as was clarified by Narusaka et al. (2021). Otherwise, the bioactivity is thought to be affected by the three-dimensional structure determined by the type of flavan 3-ol in the extension and end units of the PAC (Takanashi et al. 2017). Epicatechin pentamer strongly suppressed the gene expression of the fatty acid-binding protein 5 , involving cancer-cell invasion, in comparison with the arecatannins A2 and $A 3$, which possess catechin as the end unit. Therefore, the higher anti-IAV activity of AzPAC could be derived from the high degree of polymerization of epicatechin and the conformation that is familiar to the viral proteins. Generally, NA plays an important role in release and spread of the daughter cells from the 
host cells, but it has been shown that NA helps HA to bind to the sugar chains, thereby increasing the efficiency of infection in a recent report (Sakai et al. 2017). Considering the results of the present study and the recent report concerning virus motility, we have suggested that AzPAC binds strongly to the IAVderived membrane proteins with changes in the secondary structure of the proteins, and consequently impairs the attachment of the viral particles to the host cells.

\section{Conclusions}

In this study, we have identified the PAC components from A. zerumbet, which possess high virucidal activity against IAVs. AzPAC strongly interacted with the IAV-derived membrane proteins HA and NA. Furthermore, AzPAC changed the secondary structural rate of the a-helix and the random coil of $\mathrm{HA}$ and $N A$, which suggested that there would be a reduction in the infectious activity of the functional protein. Consequently, AzPAC prevents IAV infection by impairing the attachment of viral membrane proteins to host cells. These results will contribute to the development of drugs for the prevention of IAV infections in the future.

\section{Abbreviations}

PAC, proanthocyanidin; AzPAC, PAC from Alpinia zerumbet, ApPAC, PAC from immature apple fruit; GtPAC, PAC from green tea leaves. IAVs, influenza A viruses; HA, hemagglutinin; NA, neuraminidase; Mw, weight-average molecular weight.

\section{Declarations}

\section{Ethics approval and consent to participate}

Not applicable.

\section{Consent for publication}

Not applicable.

\section{Availability of data and materials}

All data generated or analyzed during this study are included in this published article (and its Additional files)

\section{Competing interests}

The authors declare that they have no competing interests.

\section{Funding}


This work was supported by the Research Program on Development of Innovative Technology Grants (29005AB) from the Project of the Bio-oriented Technology Research Advancement Institution (BRAIN) to T.H. and Y.N.

\section{Authors' contributions}

T.H., Y.N., and H.N. prepared molecules displaying the anti-influenza A virus from Alpinia zerumbet. T.H., Y.N., and M.N. were involved in the virucidal assays. H.M. conducted the structural analysis of PAC, a quenching assay, and CD spectrum analysis and wrote the manuscript. All authors read and approved the final manuscript.

\section{Acknowledgements}

We would like to thank Prof. Hideyuki Ito (Department of Nutritional Science, Okayama Prefectural University) for providing advice concerning the phloroglucinosis of PAC and other fruitful discussions. We would also like to thank Yuriko Imai, Chizuru Namba, Yuko Ihara, Akie Yamamoto, and Shoko Nieda of RIBS for technical assistance.

\section{References}

1. Asha K, Kumar B (2019) Emerging influenza D virus threat: what we know so far! J Clin Med 8(2):192

2. Chai WM, Shi Y, Feng HL, Xu L, Xiang ZH, Gao YS, Chen QX (2014) Structure characterization and anti-tyrosinase mechanism of polymeric proanthocyanidins fractionated from kiwifruit pericarp. $J$ Agric Food Chem 62(27):6382-6389

3. Derksen A, Hensel A, Hafezi W, Herrmann F, Schmidt TJ, Ehrhardt C, Ludwig S, Kühn J (2014) 3-Ogalloylated procyanidins from Rumex acetosa L. inhibit the attachment of influenza A virus. PLOS ONE 9:e110089

4. Edinger TO, Pohl MO, Stertz S (2014) Entry of influenza A virus: host factors and antiviral targets. J Gen Virol 95(2):263-277

5. Ghosh S, Rangan L (2013) Alpinia: the gold mine of future therapeutics. 3 Biotech 3(3):173-185

6. Hatanaka T, Narusaka M, Uraji M, Yamaji Y, Narusaka Y (2021) Identification of an anti-plant-virus molecule in Alpinia zerumbet. Bioresour Bioprocess 8:1-7

7. Howell AB, Reed JD, Krueger CG, Winterbottom R, Cunningham DG, Leahy M (2005) A-type cranberry proanthocyanidins and uropathogenic bacterial anti-adhesion activity. Phytochem 66(18):22812291

8. Hwang HS, Chang M, Kim YA (2020) Influenza-host interplay and strategies for universal vaccine development. Vaccines 8(3):548

9. Keizer J (1983) Nonlinear fluorescence quenching and the origin of positive curvature in SternVolmer plots. J Am Chem Soc 105:1494-1498 
10. Kennedy JA, Jones GP (2001) Analysis of proanthocyanidin cleavage products following acidcatalysis in the presence of excess phloroglucinol. J Agric Food Chem 49(4):1740-1746

11. Luganini A, Terlizzi ME, Catucci G, Gilardi G, Maffei ME, Gribaudo G et al (2018) The cranberry extract Oximacro® exerts in vitro virucidal activity against influenza virus by interfering with hemagglutinin. Front Microbiol 9:1826

12. Mendoza-Wilson AM, Castro-Arredondo SI, Espinosa-Plascencia A, del Refugio Robles-Burgueño M, Balandrán-Quintana RR, del Carmen Bermúdez-Almada M (2016) Chemical composition and antioxidant-prooxidant potential of a polyphenolic extract and a proanthocyanidin-rich fraction of apple skin. Heliyon 2:e00073

13. Narusaka M, Hatanaka T, Narusaka Y (2021) Inactivation of plant and animal viruses by proanthocyanidins from Alpinia zerumbet extract. Plant Biotechnol (In press

14. Narusaka M, Yamaji Y, Uraji M, Hatanaka T, Narusaka Y (2020) Inhibitory effects of Alpinia zerumbet extract against plant virus infection in solanaceous plants. Plant Biotechnol 37(1):93-97

15. Punyasiri PAN, Abeysinghe ISB, Kumar V, Treutter D, Duy D, Gosch C, Martens S, Forkmann G, Fischer TC (2004) Flavonoid biosynthesis in the tea plant Camellia sinensis: properties of enzymes of the prominent epicatechin and catechin pathways. Arch Biochem Biophys 431(1):22-30

16. Sakai T, Nishimura SI, Naito T, Saito M (2017) Influenza A virus hemagglutinin and neuraminidase act as novel motile machinery. Sci Rep 7:45043

17. Sei-Ichi K, Toda K, Matsumoto K, Ishihara C, Nonobe S, Matsunaga C, Gomi YK, Senga S, Kawaguchi K, Yamamoto A, Suwannachot J, Noda R, Kobayashi S, Hamauzu Y, Makabe H, Fuji H (2019) Isolation and characterization of a novel oligomeric proanthocyanidin with significant anti-cancer activities from grape stems (Vitis vinifera). Sci Rep 9:12046

18. Song JM, Lee KH, Seong BL (2005) Antiviral effect of catechins in green tea on influenza virus. Antiviral Res 68(2):66-74

19. Takanashi K, Suda M, Matsumoto K, Ishihara C, Toda K, Kawaguchi K, Kawahara S, Senga S, Kobayashi N, Ichikawa M, Katoh M, Hattori Y, Umezawa K, Fujii H, Makabe H (2017) Epicatechin oligomers longer than trimers have anti-cancer activities. but not the catechin counterparts Sci Rep 7:7791

20. Taubenberger JK, Morens DM (2008) The pathology of influenza virus infections. Annu Rev Pathol $3: 499-522$

21. Tsukuda S, Watashi K, Hojima T, Isogawa M, Iwamoto M, Omagari K, Suzuki R, Aizaki H, Kojima S, Sugiyama M, Saito A, Tanaka Y, Mizokami M, Sureau C, Wakita T (2017) A new class of hepatitis B and $D$ virus entry inhibitors, proanthocyanidin and its analogs, that directly act on the viral large surface proteins. Hepatology 65(4):1104-1116

22. van de Wakker SI, Fischer MJE, Oosting RS (2017) New drug-strategies to tackle viral-host interactions for the treatment of influenza virus infections. Eur J Pharmacol 809:178-190

23. Wagner R, Matrosovich M, Klenk HD (2002) Functional balance between haemagglutinin and neuraminidase in influenza virus infections. Rev Med Virol 12(3):159-166 
24. Zhang XY, Li WG, Wu YJ, Zheng TZ, Li W, Qu SY, Liu NF (2005) Proanthocyanidin from grape seeds potentiates anti-tumor activity of doxorubicin via immunomodulatory mechanism. Int Immunopharmacol 5:1247-1257

\section{Figures}

a

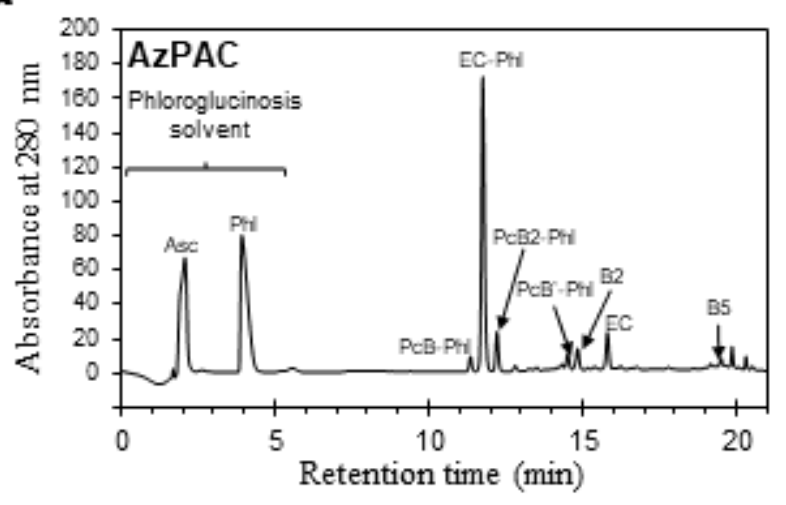

C

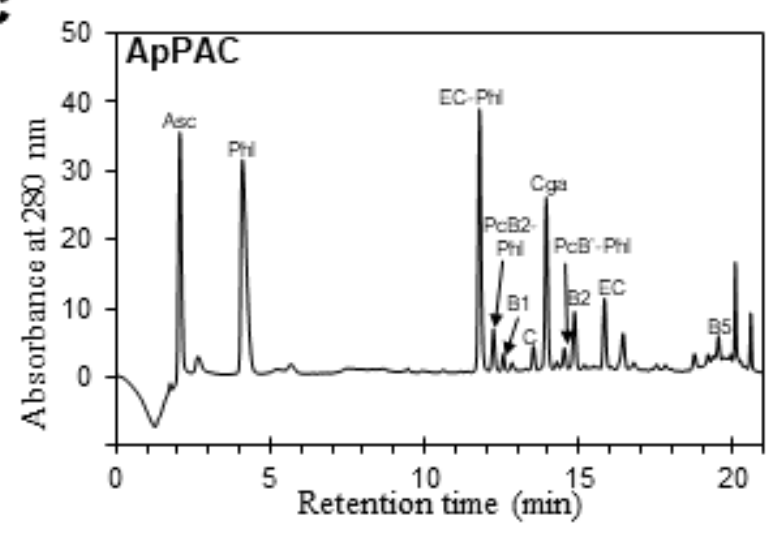

b
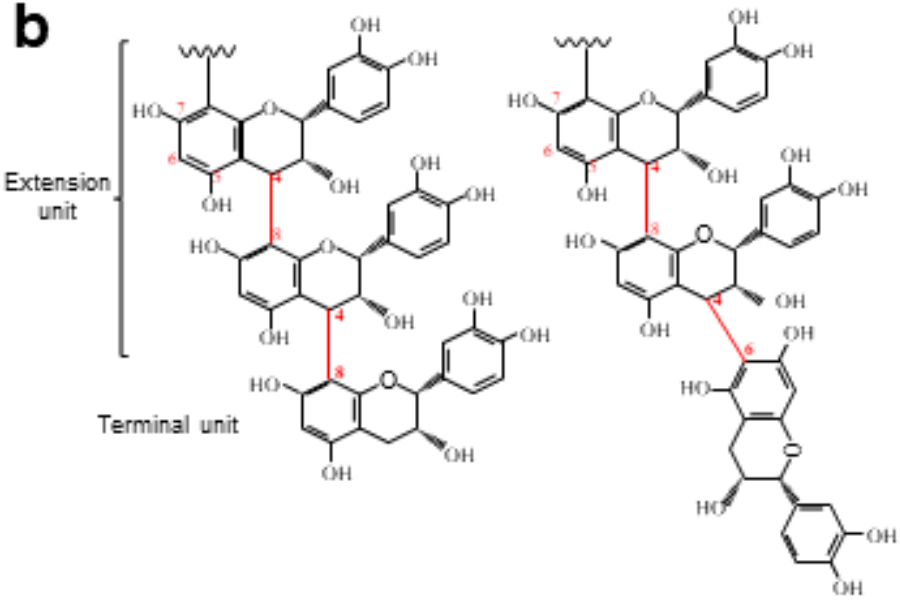

d

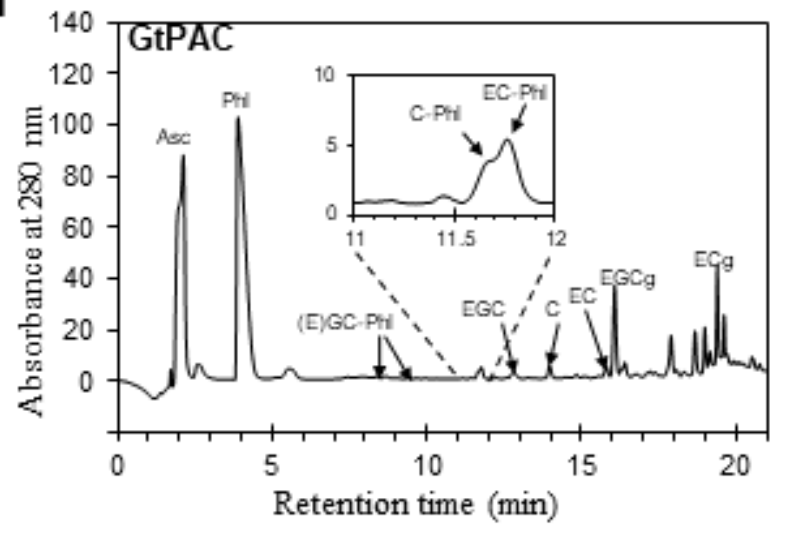

Figure 1

Characterization of the PACs that originated from three materials. Chromatograms of the extension and terminal units of the PAC extracted from (a) A. zerumbet, (b) immature apple fruits, and (c) green tea leaves after phloroglucinosis. Asc, ascorbate; Phl, phloroglucinol; PcB-Phl and PcB'-Phl, estimated procyanidin B-type-dimer adducts; $\mathrm{PcB} 2$-Phl, procyanidin B2-type adduct; $\mathrm{B} 2$ and B5, procyanidins $\mathrm{B} 2$ and B5; EC, epicatechin; C, catechin; (E)GC-Phl, (epi)gallocatechin adducts; EGC, epigallocatechin; EGCg, epigallocatechin 3-gallate; Ecg, epicatechin 3-gallate; Cga, chlorogenic acid. Undesignated peaks over 15 min of retention time are flavonoids already present in the PAC extracts. (d) Two types of AzPAC possessing different terminal unit structures. Right molecular structure shows consecutive extension units linked by $\mathrm{C} 4$ and $\mathrm{C} 8$ linkage with terminal pattern corresponding to PcB2. Left molecular structure shows consecutive extension units linked by $\mathrm{C} 4$ and $\mathrm{C} 8$ linkage with terminal pattern corresponding to PcB5. 

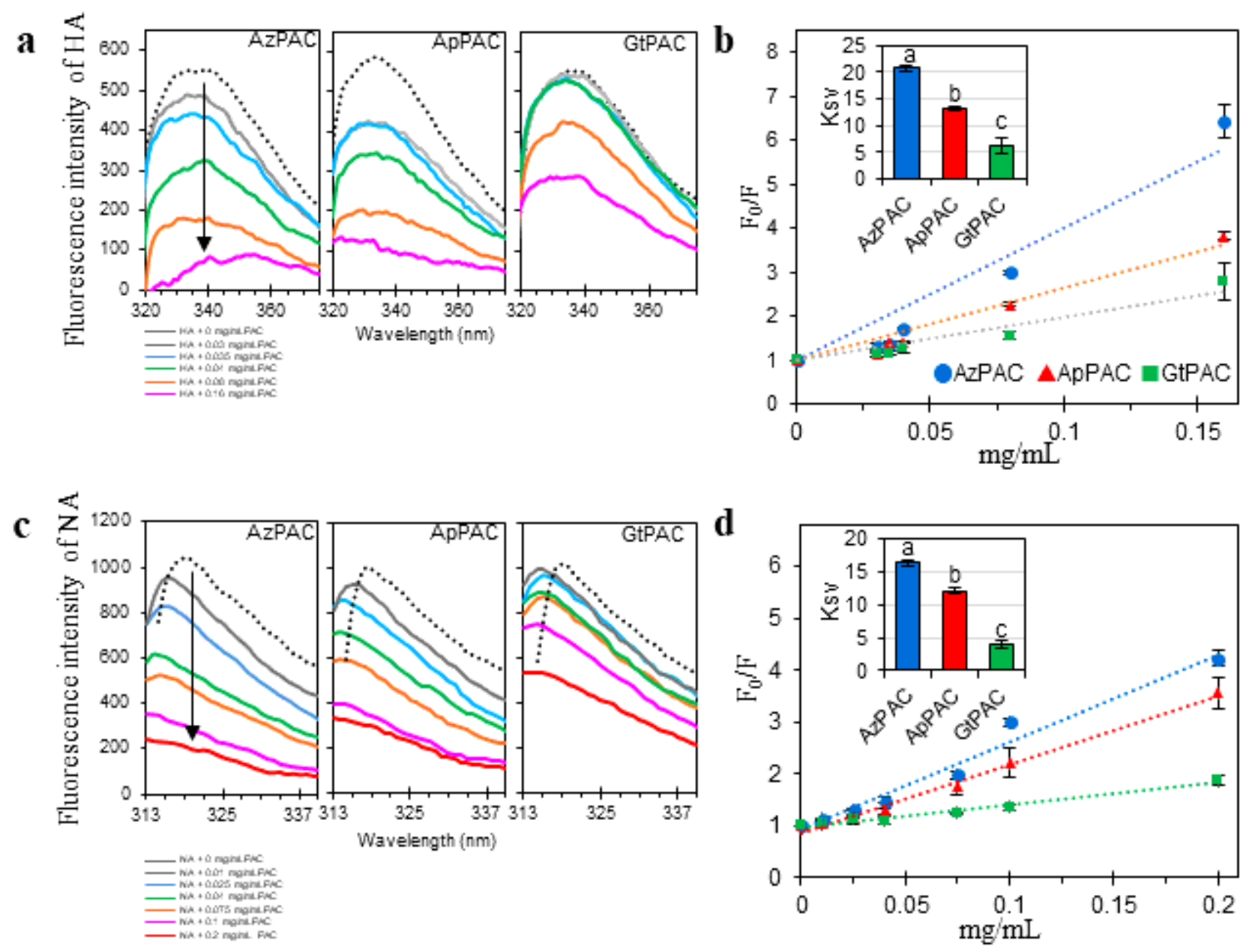

Figure 2

Quenching effects of IAV-membrane proteins in the presence of different PAC concentrations. (a) Fluorescence emission spectra (at $\lambda e x=280 \mathrm{~nm}$ ) of hemagglutinin (HA) in the presence of $0,0.03,0.035$, $0.04,0.08$, and $0.16 \mathrm{mg} / \mathrm{mL}$ of the PACs from a A. zerumbet (AzPAC), immature apple fruits (ApPAC), and green tea leaves (GtPAC). (b) F0/F Stern-Volmer plots. Binding constant Ksv values were compared among the three PACs (an inset). The results show standard errors ( \pm SE) of three replications for each PAC. Different alphabetical letters show the differences among the three PAC products by a Tukey's multiple comparison test $(P<0.05, n=3)$. (c) Fluorescence emission spectra (at $\lambda e x=285 \mathrm{~nm}$ ) of Neuraminidase (NA) in the presence of $0,0.01,0.025,0.04,0.075,0.1$, and $0.2 \mathrm{mg} / \mathrm{mL}$ of AzPAC, ApPAC and GtPAC. (d) F0/F Stern-Volmer plots. Binding constant Ksv values were compared among the three PACs (an inset). The results show standard errors ( \pm SE) of three replications for each PAC. Different alphabetical letters show the differences among three PAC products by a Tukey's multiple comparison test $(P<0.05, n=3)$. 

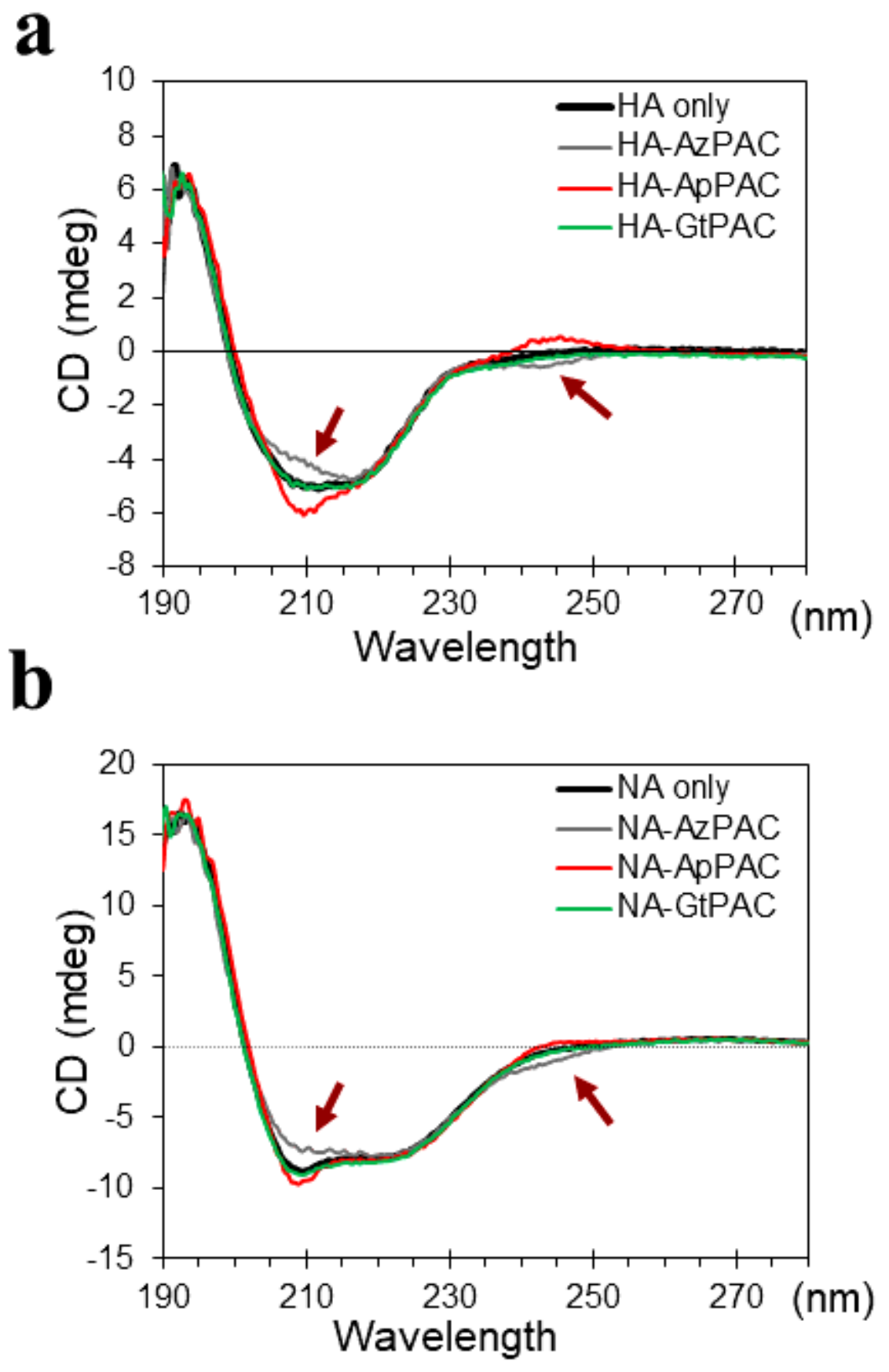

Figure 3

Interactions of the PACs with viral membrane proteins using the circular dichroism method. Circular dichroism (CD) spectra of HA (a) and NA (b) in the presence of AzPAC, ApPAC and GtPAC. The arrows show the positions where the $C D$ spectra changed remarkably.

\section{Supplementary Files}

This is a list of supplementary files associated with this preprint. Click to download. 
- GraphicalAbstract211013.pptx.tif

- SupplementalFigure211013.tif 